\title{
A New Therapeutic Community: Development of a Compassion-Focussed and Contextual Behavioural Environment
}

\author{
David Veale, ${ }^{1 *}$ Paul Gilbert, ${ }^{2}$ Jon Wheatley ${ }^{1}$ and Iona Naismith ${ }^{1}$ \\ ${ }^{1}$ Institute of Psychiatry, King's College London and the South London and Maudsley NHS Foundation Trust, \\ London, UK \\ ${ }^{2}$ Mental Health Research Unit, Kingsway Hospital, Derby, UK
}

\begin{abstract}
Social relationships and communities provide the context and impetus for a range of psychological developments, from genetic expression to the development of core self-identities. This suggests a need to think about the therapeutic changes and processes that occur within a community context and how communities can enable therapeutic change. However, the 'therapeutic communities' that have developed since the Second World War have been under-researched. We suggest that the concept of community, as a change process, should be revisited within mainstream scientific research. This paper briefly reviews the historical development of therapeutic communities and critically evaluates their current theory, practice and outcomes in a systematic review. Attention is drawn to recent research on the nature of evolved emotion regulation systems, the way these are entrained by social relationships, the importance of affiliative emotions in the regulation of threat and the role of fear of affiliative emotions in psychopathology. We draw on concepts from compassion-focussed therapy, social learning theory and functional analytical psychotherapy to consider how members of a therapeutic community can be aware of each other's acts of courage and respond using compassion. Living in structured and affiliative-orientated communities that are guided by scientific models of affect and self-regulation offers potential therapeutic advantages over individual outpatient therapy for certain client groups. This conclusion should be investigated further. Copyright $\odot 2014$ John Wiley \& Sons, Ltd.
\end{abstract}

Key Practitioner Message:

- Current therapeutic community practice is not sufficiently evidence based and may not be maximizing the potential therapeutic value of a community.

- Compassion-focussed therapy and social learning theory offer new approaches for a therapeutic environment, involving an understanding of the role, nature and complexities of compassionate and affiliative relationships from staff and members, behavioural change guided by learning theory, a clear formulation based on threat-derived safety strategies, goal setting and positive reinforcement.

Keywords: Therapeutic Community, Attachment, Compassion, Functional Analytical Psychotherapy, Reinforcement, Systematic Review

\section{INTRODUCTION}

Like other mammals, humans evolve and live within communities, family and kin groups. Indeed, the social dynamics of group living have been fundamental to the evolution of a number of human competencies such as self-awareness, cooperation, social sharing and capacities for mentalizing, empathy and affect regulation (Baumeister \& Leary, 1995; Dunbar \& Barrett, 2007). The evolutionary pressures that gave rise to these competencies are often

*Correspondence to: David Veale, Institute of Psychiatry, King's College London and the South London and Maudsley NHS Foundation Trust, London, UK.

E-mail: david.veale@kcl.ac.uk referred to collectively as the social brain hypothesis (Dunbar, 2007, 2010). There is increasing evidence that individuals adapt their behaviour and experience of the self according to the social context in which they operate-for good or ill (Cacioppo \& Patrick, 2008). Different ecologies give rise to different values, senses of self and strategies involving trust and loyalty (Li, 2003). Gilmore (1990) offers many examples of how self-identities are created within community contexts, such that male identity and behaviours can become aggressive and violent in some social contexts yet are benign in others. Group pressure and the desire to conform can lead to all kinds of immoral behaviours that are destructive, such as committing atrocities in war or youths getting caught up with criminal gangs (Gilbert, 
2005, 2009; Kelman \& Hamilton, 1989; Zimbardo, 2008). Even when people appear to be behaving according to the requirements of the group, it is not always clear whether this is submissive behaviour or even cynical compliance rather than an internalized valued system.

Social relationships and social context, especially affiliative and caring ones, play major roles in physical health (Cacioppo \& Patrick, 2008; Holt-Lunstad, Smith, \& Layton, 2010) and profoundly influence neurophysiology (Cozolino, 2007) and genetic expression (Belsky \& Pluess, 2009). There is even evidence that gene expression is influenced by a change in the dynamics of group living, such as social status changes (Tung et al., 2012). We offer these preliminary concerns to indicate the power social relationships and communities can wield over their members, which has been put to therapeutic use in what are called therapeutic communities (TCs). (When using the term 'member', we refer to both the clients or residents and the staff.) We will argue that current TCs have operated largely outside mainstream psychological research since the Second World War and that a new generation of TCs, informed by such research and guided by a compassion-focussed and contextual behavioural environment, offers potential. However, this approach obviously requires further conceptual and outcome research. In this article, we will describe the following:

1. The history of the TCs to understand the context

2. The theory behind contemporary TCs

3. A critical evaluation of the processes in TCs

4. A systematic review of outcomes of TCs

5. An evolutionary and compassion-focussed approach for a new approach to a TC

6. The contribution of social learning theory and functional analytical psychotherapy (FAP) to a new TC

7. The need for structured activity, goals and values in a new TC

8. The new TC in action

\section{THE HISTORY OF THE THERAPEUTIC COMMUNITIES}

Psychodynamic therapists have defined a TC as 'a consciously designed social environment and program within a residential or day unit in which the social and group process is harnessed with therapeutic intent. In the TC, the community is the primary therapeutic instrument' (Roberts, 1997). Membership is clearly defined, and the staff has a facilitative role in operating the community as mediators of change. The members have significant involvement in decision-making and the practicalities of running the unit. Their life together is configured to help members develop personal responsibility, build an understanding of themselves and change their ability to regulate both emotions and behaviour (Kennard, 1998). The process is one of dynamic, reciprocal interactions where individuals receive feedback and support in the change process. They are engaged in a range of activities including community meetings, group psychodynamic therapy, social interaction and communal living. Some communities may include individual psychotherapy, but it is the relationships between members and the community that are regarded as the mediators of change. The term 'therapeutic community' does not therefore refer simply to a place for healing-it is used to describe a community where the relationships between the members (including staff) and with the community are reflected upon in group therapy.

Therapeutic communities were traditionally residential. Economic considerations have meant that the large majority of adult TCs are now day centres for which the members usually attend 3-5 days a week, for between 6 and 24 months. TCs exist in various settings, including adolescent, adult mental health and learning disability units and prisons. Populations served include those with severe personality disorder and alcohol or substance abuse.

Previous literature (Borthwick et al., 2001; Kennard, 1998; Whiteley, 2004) has described the history and evolution of the first generation of TCs, beginning with Tuke's 'moral therapy' at the York Retreat in 1796. This involved the minimum use of restraint, early forms of behaviour therapy (including activity scheduling) and a humane and caring environment. The medical historian Roy Porter (2002) says that William Tuke, a tea merchant, modelled the retreat on bourgeois family life. Patients and staff lived, worked and dined together in an environment where recovery was encouraged through praise and rewards rather than punishment, the goal being the restoration of self-control. Tuke's grandson Samuel noted that medical therapies had initially been tried there with little success; 'the Retreat had then abandoned "medical" for "moral" means, kindness, mildness, reason and humanity all within a family atmosphere-with excellent results' (Porter, 2002, p. 104).

The basic psychology was a form of benevolent paternalism guided by Quaker beliefs for those who had 'lost their reason' (Borthwick et al., 2001). 'Compassion and kindness' was the basis of this movement (Ballatt \& Campling, 2011). This focus was something of an innovation, although in keeping with the times that saw a resurgence in concerns of compassion and social justice throughout Europe, with notable figures such as Philippe Pinel (1745-1826) also attempting to introduce more humane care in various asylums in France (Porter, 2002). This was to change with the Second World War. A second wave of TCs was configured for soldiers needing to share the experience of war trauma in all its forms. Now the focus moved from helping people who had 'lost their reason' experience a compassionate family environment towards enabling traumatized soldiers to come to terms with their experiences and return to fighting. The capacity to get on with others was 
stressed because soldiers need this attribute in order to remain effective. The importance of this legacy derives from the original focus-working with the angry, frightened and traumatized soldier to try to (re)build his sense of responsibility to others and his ability to deal with feelings of aggression to others who may well be comrades. Groups needed members who were affiliative and corporative within the group, but killers without.

\section{THE THEORY BEHIND CONTEMPORARY THERAPEUTIC COMMUNITIES}

After the war, a second generation of TCs began to take shape. Individuals from more diverse backgrounds with different types of problem were considered potential candidates for TCs. This work progressed at the Cassel Hospital in London and then at the Henderson Hospital in Surrey (Jones, 1956), still guided by psychoanalytical group therapy fashioned from war experiences (Main, 1946). Rapoport (1960) identified a number of defining features of a TC at the Henderson Hospital, which were updated by Haigh (1999). The first characteristic of TCs is 'permissiveness', meaning that members were expected to tolerate a high level of expressed emotion. For example, members may be encouraged to verbally express their anger and to be 'authentic' in their emotions. The rationale was of catharsis and to allow members to acknowledge their 'true' feelings. TCs are structured as communal living experiences with clear boundaries and rules, support for members expressing emotion and frequent group-meetings. This is linked to the theoretical principle of 'containment' and the developmental stage of 'being held' by one's parents when one is distressed.

A second characteristic was 'communalism' and group living: close relationships, sharing of facilities (e.g. a dining room) and free communication were encouraged, in order to enable members to learn from one another in groups and everyday life. Everything may be brought to the group so that any out-of-hours contact or communication between members is available for discussion in the community meeting to avoid 'splitting' between members (whereby some individuals develop polarized views of another member because they have had very different interactions with the member). This is linked to the developmental stage of play and speech and development of a sense of self as separate.

A third characteristic was 'reality confrontation', whereby members confront each other's behaviour and its consequences in the 'here and now'. The culture in the community is of 'living-learning' whereby members learn about themselves by reflecting on daily life events. Typically, there are daily community meetings, after which the staff debrief, provide interpretations and reflect on the relationships between themselves and the members. This is linked to the developmental stage of finding a place among others.

The fourth feature was democracy and de-institutionalization. A TC was equitable and non-hierarchical, and members were actively involved in decision-making for their own and others' care. This is now known as a 'culture of empowerment' (Campling \& Haigh, 1999). The theory is that sharing in decision-making helps build self-confidence and a sense of responsibility. Note that this is not the same as a 'family environment', the focus of the earlier efforts of the Tukes, but a stage of establishing one's self as a seat of action. Some TCs were subsequently recognized as fully democratic, requiring voting procedures for all community affairs (including the admission, care and discharge plans of members). Those that were regarded as modified TCs were not fully democratic.

\section{A CRITICAL EVALUATION OF THE PROCESSES WITHIN THERAPEUTIC COMMUNITIES}

The practice of second-generation TCs is guided by whether they achieve agreed standards obtained by expert opinion and consensus of members rather than by process-based research. TCs thus try to raise standards by peer accreditation according to the defined values (Royal College of Psychiatrists, 2010). Many of the processes are strongly shaped by group psychodynamic theory reaching back to the war experience rather than the original Tuke retreat or evidence from current psychology. We discuss below our concerns that some TC practice draws upon processes of group belonging without sufficient consideration of when such processes may be unhelpful.

In this section, we critically evaluate the processes within second-generation TCs-we identify some of the processes that are consistent with promoting the principle of feeling 'safe'. We will argue that this is one of the most important outcomes for a member of a TC and that it is safeness, the provision of 'a secure base and safe haven', that opens attention and capacities for encouraging exploration and also regulation of difficult emotions-as originally envisioned by Bowlby (1969, 1973; Mikulincer \& Shaver, 2007). For most animals and humans (and we suggest members of a TC), safeness is best achieved by reducing the signals of interpersonal threat and increasing affiliative signals and capacities (Depue \& Morrone-Strupinsky, 2005; Porges, 2007; Taylor, 2006). We will discuss how a major way humans do this is by sharing and cultivating compassion (e.g. with caring interest and empathic engagement with distress) to self and others (Gilbert, 2010). 


\section{Attachment}

Attachment theory has now been integrated into many psychotherapeutic approaches (Danquah \& Berry, 2013; Wallin, 2007). The model behind TCs is also of attachment theory. Attachment theory stresses attachment to a reliable and powerful dominant other, usually a mother figure, possibly a father or 'significant other' (Bowlby, 1969, 1973, 1980; Mikulincer \& Shaver, 2007). Bowlby was particularly concerned with the behavioural aspects of care. The first is a tendency for the infant to seek proximity to a caring other. Second is the ability of the caring other to act as a safe haven who regulates threat exposure for the infant, keeping the infant out of harm's way, chasing off predators or picking up the infant and bringing it back to stay close and being soothing of the infant's distress (all protective functions). Third was acting as a safe/secure base, from which the child gains the confidence to go out, explore and develop independence. Fenney and Thrush (2010) suggest that a secure base operates in adult relationships with the functions of encouraging exploratory behaviour and facilitating confidence and self-development and is best developed in the context of safeness. For these functions, Fenney and Thrush suggest that caring others should be available and non-interfering and should encourage and reward efforts.

The importance of an accessible and available benevolent 'authority', who is the source of those functions, is crucial in attachment theory, and indeed, a hierarchy can have a very containing and protective function. Familybased attachment was at the centre of Tuke's TCs.

Many theorists believe that early trauma can disrupt the smooth integration and operation of the attachment systems and in consequence produce a whole range of potentially maladaptive defences to threat (Van der Hart, Steele, \& Nijenhuis, 2006). A helpful description of this process is given by Liotti and Gumley (2008):

Attachment theory explains the origins of disorganized attachment behaviour in terms of conflict between two different inborn systems, the attachment system and the fight-flight (i.e. defence) system. The attachment and defence systems normally operate in harmony (i.e. flight from the source of fear to find refuge near the attachment figure). They, however, clash in infant-caregiver interactions where the caregiver is at the same time the source of, and the solution for, the infant's fear... Being exposed to frequent interactions with a helplessly frightened, hostile and frightening, or confused caregiver, infants are caught in a relational trap: their defence system motivates them to flee from the frightened and/or frightening caregivers, while at the same time their attachment system motivates them, under the influence of separation fear, to approach them. Thus, the disorganized infant is bound to the experience of 'fright without solution'... This experience may be understood as a type of early relational trauma, which exerts an adverse influence on the development of the stress-coping system in the infant's brain (Liotti \& Gumley, 2008, p. 118).

Early close-attachment experiences influence interpersonal styles of relating and, when they function poorly, can create individuals who may be avoidant, demanding, distrustful or exploitative of others (Mikulincer \& Shaver 2007; Wallin, 2007). The theory is that these responses will gradually be corrected by self-observation and feedback from other members of a TC, and in a TC, members can be motivated to engage in these processes through a desire for a sense of belonging to the community. TCs make the assumption that the community will act as the 'attachment object', where individuals will seek proximity to the group rather than avoidance and will be able to use a group as a safe haven and a secure base. However, there is an additional element, which is not particularly related to attachment theory. In current TCs, there is a focus on a sense of belonging to the group and peer-group attachments, which may be better regarded as affiliative psychology. Baumeister and Leary (1995) note that for interpersonal relationships to be successful, there is a need for frequent, affectively pleasant interactions with a few other people and, second, these interactions must take place in the context of a temporally stable and enduring framework of affective concern for each other's welfare.

It is the desire to obtain security from belonging to a group that encourages individuals to change their behaviour in order to conform and feel part of the group and avoid rejection and sanction from not doing so. However, it is important not to mix functions of the provision of safeness and confidence with the issue of social conformity. For example, these social processes can be used for good or ill. They are prominently used in cults that make belonging core to their process (Zimbardo, 2008). People can do immoral things for such reasons, and indeed, conformity does not always stem from feeling safe and attached in groups but rather feeling threatened (Kelman \& Hamilton, 1989).

As in cults and religious communities, members in a TC are expected to care for and emotionally support one another and to be 'held' in mind by other members. There is a culture of belonging within the community, with formal structures for referrals, joining and leaving (Haigh, 1999). Formal structures for referral, joining and leaving a TC will encourage a sense of belonging to a community and make it easier to tolerate the distress of loss and encourage the maximum degree of responsibility. These formal structures are in our view helpful processes in modelling for people who have had inconsistent and chaotic interpersonal relationships.

Pearce and Pickard (2013) suggest that the role of belongingness is central to how TCs work. They recognize 
however that it is not a requirement that the environment of TCs is 'affectively pleasant' (Baumeister \& Leary, 1995) but that mutual concern is an aspiration of all TCs. We think that a better term for belongingness is 'connectedness to others' and that connectedness needs to be encouraged to occur to other members rather than attachment to the community. Attachment to a community will depend on shared values and tasks and is not the same as feeling connected to other members. We also suggest that affiliative emotions and caring for others should not just be focussed on group members but cultivated as a process for the relationship to one's self and to others in general (outside the community). We will discuss later how the feeling of connectedness can be best cultivated using a compassion-focussed and social learning model.

\section{Creating the Community}

In a TC, the focus is on relationships between members and the responsibilities to the community as a whole, which is regarded as the 'attachment' object. Although attachment theory has been incorporated into ways of thinking about TCs, the older themes of interpersonal relationships, e.g. developing affiliations between individuals and learning how to deal with aggression and anger, are as much the key textures of TCs now as they were 60 years ago. What started as a wartime effort to get soldiers back to the front, guided by early psychodynamic concepts, gradually evolved and progressed into a complex array of approaches guided by different theories and philosophies (Campling \& Haigh, 1999). Indeed, some are guided by general philosophical orientations to life rather than research into the psychological processes of change, with ideas that everybody will benefit from the same kind of 'caring behaviours' (Tucker, 1999) and democracy.

Democracy, participation and de-institutionalization, now known as a 'culture of empowerment', are some of the characteristics of psychodynamic TCs. TCs were thus pioneers in the principle of user involvement and taking responsibility. The principle of shared decision-making and user involvement now occurs in all health care in varying degrees of dilution. The ability to influence one's environment is likely to influence the quality of a member's experience of a community and is especially important if a member will be around for a year or more. The rationale is that it allows healthy parts of the personality to emerge. We agree that taking responsibility is very important, but there is no evidence that democracy or lack of hierarchy is a powerful mediator of change for everyone. We would question the 'broad-brush' approach to democratic processes: we believe that there should be consideration of an individual's formulation, including predictions on how an individual interacts with others in a community. This should be shared in the community so that others can provide feedback and provide opportunities to explore and shape behaviours that are less developed. For example, an avoidant and unassertive member might benefit from taking a more active leader-like role in running the community. A member who finds it easy to use (or possibly abuse) power should be participating less in running the community and be more other and helper focussed (i.e. positions should be given to those who would benefit most from them). Pearce and Pickard (2013) also emphasize that TCs work by the unique combination of belongingness and responsible agency. Bizarrely, they suggest that cognitive-behavioural therapy is cautious about introducing the language of responsibility and is careful to preserve a non-judgemental attitude. This appears to be a straw dog argument that we would strongly disagree with. We shall discuss a model that is non-judgemental and promotes the outcome of responsible agency by the process of promoting acts of courage by positive reinforcement and compassion.

Another characteristic of TCs was of 'permissiveness' and 'reality confrontation', in which members may have a high degree of expressed emotion. As noted above, the issue of aggressiveness was central to the early therapist working with war-traumatized soldiers. Therefore, some individuals who are avoidant of emotion learnt they could express themselves safely, and this is very helpful. However, there is no evidence that activating the threat system is helpful for other members in a TC. Indeed, facilitating open expression of anger could, for some patients, simply be a rerun of family scenes of anger (e.g. from a parent) that generated fear in them, when in actual fact what the child actually needed was parents who were emotionally controlled or someone who would protect them from 'the anger'. Indeed, as noted above, protector functions are very important for children and, we suggest, for some individuals in certain states of mind. So, open expression of anger could actually create the conditions for disorganized attachment in individuals whose attachment systems are very fragile. Unfortunately, there is very little research here in including how members in a TC actually experience these encounters with other members who are threatening.

Another concern is that some people with borderline personality disorder may have an under-regulation of emotion. The problem here is that anger may not be an authentic feeling as such but rather defensive and/or a cover for authentic feelings. Aggression can be a cover for avoidance of traumatic memories, fear or intense grief and yearning. Encouraging, or at least not discouraging, anger (as affect) regulation may simply encourage experiential avoidance (e.g. of hurt or grief). So although it is true that people who are fearful of emotions, be it anger, anxiety or grief, will need to learn to tolerate it via exposure, the way in which exposure is conducted and 
in particular the audience on whom it impacts need careful thought. Experienced therapists know this of course, but it is poorly researched and articulated in the literature. Different people fear different emotions, e.g. depressed people can be fearful of anger; yet for others, this is not so, and it is sadness, anxiety, yearning for love or even guilt/remorse that is blocked. For many who are quite comfortable with anger as a defensive first-line response, it is a powerful form of avoidance. It is important to identify in a formulation the patterns of emotions and behaviours that are overused defensively and those that are avoided and to respond to the latter with behavioural experiments. This is no different in principle to the behavioural experiments or exposure that is now standard for anxiety interventions.

Interestingly, research into mentalizing focusses on these kinds of processes where individuals are not able to understand or reflect on their emotions or understand that one emotion can be a function of attempting to achieve safety from another (Allen \& Fonagy, 2007). If reflective function is one of the aims of therapy, then we need to understand that the psychology that facilitates it is most effectively delivered in a relatively safe environment, not a highly charged one (Liotti \& Gilbert, 2011). The lack of an individualized formulation might lead to a general assumption in staff that any expression of a strong emotion by a patient is to be encouraged, but this does not take into account the function of the emotion. Thus, adaptive expression of any emotion may be regarded as important regardless of function.

Thus, a key concern is that an environment of high emotional expression within a TC may undermine other members' feelings of safeness, especially if they come from emotionally charged families who generated a lot of fear in them (Liotti \& Gumley, 2008). Staff may believe that an instance of high emotional expression is contained within the boundaries of the community and indeed report this in staff meetings. However, they do not formally evaluate containment, safeness or connectedness to others. A TC may be merely reproducing the high-level emotional expression that members or staff are used to during childhood and do little to enhance connectedness. The question should always be 'does this intervention increase connectedness and safeness for a member and facilitate the maturation process associated with safeness?' It is safeness and 'a secure base' that open attention and capacities for encouraging acts of courage in exploration or connectedness and also regulation of difficult emotions-as originally envisioned by Bowlby (1969). For most animals, humans and members of a TC, safeness is best achieved by reducing the signals of threat and increasing affiliative signals and capacities. One major way humans do this is by sharing and cultivating compassion (e.g. empathic engagement with distress and with caring interest) to self and others (Gilbert, 2010) and by the use of positive reinforcement when members act towards their goals with courage.

\section{Boundaries}

An important aspect of creating safeness is to have boundaries. Some institutions emphasize boundaries without focussing on the motivation behind them. The motivation seen in some settings can be for obedience to authority in which members have to know that certain behaviours are unacceptable even if the authority is the so-called group. This type of obedience can be seriously problematic (Kelman \& Hamilton, 1989). However, TCs have rightly highlighted how boundaries are important if members are to feel safe and contained. A loving parent puts down boundaries to help and protect the child, whereas the authoritarian uses boundaries to be respected and maintain a sense of authority and power. It is important for a TC to be especially psychologically minded for members to understand the difference, especially given the likely abusive backgrounds of many members. Hence, clarity on the motivation and function behind boundaries needs to be part of the process of setting boundaries. The non-verbal behaviour in these contexts is likely to be crucial, and staff will need to be trained in this. It may be too easy for staff's anxieties and (unconscious) authoritarian traits to be stimulated in these contexts (Zimbardo, 2008). Again, there is little research on user experiences to say how common this may or may not be within a TC or the degree of influence that the clients have in setting boundaries.

The concept of the TC evolved in the context of help for substance and alcohol misusers (De Leon, 2000). Phoenix House and Daytop communities originally developed in the USA and overlapped to some degree with adult TCs. Concept TCs are not democratic but have a hierarchical structure in which an individual works to obtain privileges and responsibility. There is greater stress on members acknowledging their addiction and being able to identify with others with the same problem. Such groups are more likely to use confrontation and shame, which we discuss further below.

The combination of high expressed emotion and 'reality confrontation' means there may be a sense of edginess and tension within a TC. Outbursts of anger are not viewed negatively: the community is judged on both how it contains the anger safely and how it provides a safe place to be angry. The risk is that members may be confronted for their disruptive behaviour and can be criticized or shamed, with others in the community expressing their annoyance, upset, disappointment or anger. Unfortunately, 'shaming and blaming' may mirror the early experiences of anger and disappointment familiar to that individual and do not enhance a feeling of safeness and 
connectedness to others. This is especially true if the staff do not understand the distinctions between shame and guilt (Gilbert, 2007, 2011; Tangney \& Dearing, 2002). Guilt and the ability to experience remorse is indeed very important in developing prosocial relationships and is very different to shame. The responses of staff and members may therefore be experienced by the individual as invalidating and may be seen as evidence to confirm their negative self-beliefs. Being confronted by other members or staff in the group about problematic behaviour may place too much 'heat' on the individual, with the result that they feel overwhelmed by intense emotion, particularly shame, that might actually make it harder for them to reflect on their behaviour. Opportunities for more protected reflection time, which may facilitate insight in 'harms caused', and remorse, sadness and guilt may be more helpful, and here, one could take a leaf out of the work on restorative justice procedures (e.g. Zehr, 2002).

There is a risk that shame (rather than guilt) may be used in an attempt 'to make' members more aware of the impact of their actions on the community as a whole and regarded as a therapeutic process. This occurs particularly in concept TCs and prisons, where it is believed that shame alters moral behaviour despite all the evidence that shame, in contrast to guilt, does not (Dearing \& Tangney, 2011). Most researchers now recognize shame as a highly self-focussed emotion that stimulates threat and defensive manoeuvres. These manoeuvres can vary from anxiety and submissive behaviour through to denial and avoidance and onto aggressive counterattacks (Gilbert, 2007; Tangney, Wagner, Hill-Barlow, Marschall, \& Gramzow, 1996). Guilt, in complete contrast, requires a very different process of engagement. It is focussed more on reparative behaviour rather than an evaluation of a global sense of self (Tangney \& Dearing, 2002) and on the ability to be empathic to the harm that we do and be in touch with sadness and sorrow. If we cannot deal with our own sadness and sorrow, possibly arising from the hurts we have experienced, then we may find it very difficult to deal with the sadness and sorrow that arises when we hurt others (Gilbert, 2009, 2010). We know of no data that show that emotional expression groups are always the best way to deal with 'reality confrontation'. We suspect it is easy for vulnerable members to confuse conformity with genuine moral development. Reparation efforts based on guilt are quite different to reparations based on shame, and in some ways, this links to research into retributive (shame-focussed) and restorative (guilt-focussed) justice (Gilbert, 2009, 2011). It may be better to use very small groups with only members involved in a conflict to discuss the issues compassionately and reduce the risk of shame, i.e. borrowing insights from the restorative justice movement (e.g. http://www. restorativesolutions.org.uk) rather than involving the whole community. This needs to be tested empirically in terms of the outcome of safeness and connectedness to others.
After each community meeting or group therapy in a psychodynamic TC, the staff will debrief, provide interpretations and reflect on the relationships between themselves and members. This can be costly in terms of staff time. The process of psychodynamic reflection after each group has not to our knowledge ever been evaluated as a means of promoting safeness and connectedness between members. Although it is important that staff spend time in supervision or reflection to understand behaviour of members, it may be more clinically and cost-effective to spend the bulk of the time face to face with members-providing a safe haven, modelling and responding in the moment and shaping acts of courage in achieving their goals. Again, this could be tested empirically to determine which strategy is more effective.

\section{EFFECTIVENESS OF THERAPEUTIC COMMUNITIES: A SYSTEMATIC REVIEW}

Taken as a whole, the effectiveness of TCs has a thin evidence base for a range of problems. For example, TCs are not recommended in treatment guidelines for borderline personality disorder (National Institute for Health and Clinical Excellence, 2009). To identify relevant past systematic reviews, a search was conducted on the PsycINFO database that yielded 19 results. Lees, Manning, and Rawlings' (2004) study was the most up-to-date research that involved a range of clinical populations and psychological symptomatology outcome measures. They located eight randomized controlled trials (RCTs) and 21 other studies using a control group, which had clear outcome criteria, raw data that were reported and a clear specification of the original sample before attrition. These 29 studies were included in the present review. A new systematic review was then conducted to try to identify additional, particularly more recent, studies that searched on 'Therapeutic Community' or 'Therapeutic Communities' and 'randomized controlled trials' or 'RCT'. This yielded eight results on PsycINFO and 82 on MEDLINE. From these results, studies having the following characteristics were selected: (i) comprised of a matched-control trial or RCT and (ii) compared TC with a different type of treatment (rather than no treatment or early discharge from the TC). These criteria are more stringent than the ones used by Lees et al. (2004) who permitted matchedcontrol trials comparing dropouts and completers from the same TC; thus, we do report below on some such studies that were identified by Lees et al. (2004). We did, however, include studies where the control condition was standard incarceration, or treatment that was less frequent than the TC programme; yet such studies also have clear methodological limitations since it is possible that a non-TC treatment of similar frequency could produce equal or better outcomes. Where other systematic reviews 
of relevant RCTs and matched-control studies were identified, their results were also included. The 90 identified results were checked against our inclusion and exclusion criteria as outlined by Figure 1, resulting in seven to be analysed alongside the 29 identified by Lees et al. (2004).

The vast majority of the identified studies relate to substance misuse and/or forensic services, including six of the eight RCTs identified by Lees et al. (2004). There is some (limited) evidence for secure TCs being superior to standard prison services (in terms of reducing risk of future offending behaviour) and very little evidence for TCs being superior to standard treatment for substance abuse. Furthermore, the evidence that does exist comes from studies with serious methodological limitations: all studies of forensic or substance misuse TCs included in this review used either no-treatment control groups or control treatments that differed considerably on intensity, length and other factors. For example, Sacks, McKendrick, and Hamilton (2012) compared a prison TC programme ( $20 \mathrm{~h} /$ week for 6 months) and a cognitive-behavioural intervention (6-8 h/week for 16 weeks). Several authors of systematic reviews have drawn the same conclusions (Fiestas \& Ponce, 2012; Perry et al., 2009; Smith, Gates, \& Foxcroft, 2006). This evidence base, of course, has limited applicability to the treatment of clients with personality disorders in non-forensic settings, given the differences both in client group and in environment (e.g. security issues in forensic settings necessitate some hierarchy between staff and prisoners). Thus, there is a very poor evidence base for TCs that do not focus on forensic or substance use populations. The majority that exist compare completers of TC treatment programmes with dropouts of the same programmes or no-treatment controls, which predictably find that TC treatment is better than no treatment and that longer durations of treatment (9-12 months and above) were superior to shorter durations.

Only three RCTs involving non-forensic or substance misuse services were located, and results overall did not support the superiority of TC treatment over other treatments. Lehman and Ritzler (1976) compared psychiatric inpatients admitted to either a TC or a service implementing a medical model and found the former to have higher patient satisfaction but greater readmission rates (26\% versus 19\%). Hansen and Slevin (1996) compared a standard psychiatric ward to a ward informed by TC values (including greater patient involvement in treatment, group therapy focussed on emotional expression and regular community meetings). The wards were matched on patient number, treatment duration and patient diagnoses (primarily schizophrenia). The TC-style ward was found to be significantly higher in patient

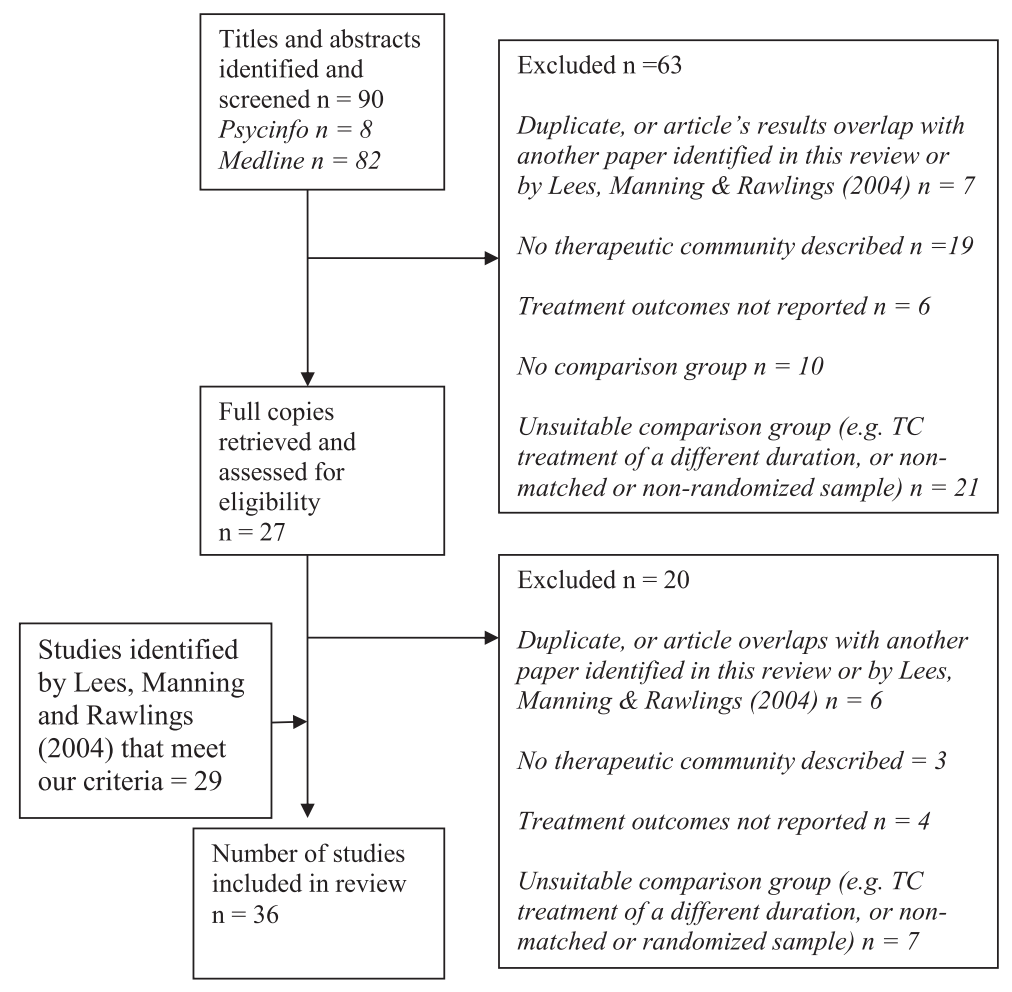

Figure 1. Process for systematic review of therapeutic communities 
involvement, support and practical orientation; however, no comparison was made in terms of symptomatology or outcome. Finally, Craft, Stephenson, and Granger (1964) compared male delinquents in two non-secure settings: one a TC and the other an authoritarian disciplinary programme with individual treatment. In a direct challenge to the effectiveness of TC treatment, the traditional unit produced significantly lower rates of reconviction and significantly lower numbers of clients still needing institutional care.

In summary, the evidence base does not support the claim that TCs are superior to other interventions. There is a paucity of evidence for mental health and personality disorder populations. The evidence base for forensic and substance abuse populations is minimal, and the studies that have been conducted consistently have significant methodological limitations. Typically, the control condition is standard incarceration or involves treatment of lower frequency than that delivered by the TC. Furthermore, TCs are complex interventions, and crucially, there are no studies on the supposed mediating factors in a TC. There is no basic science to determine, for example, whether permissiveness or reality confrontation increases safeness or belongingness or responsibility for change. TCs are however still being commissioned by purchasers for a range of care. Current TCs are relatively expensive to deliver, and there are no studies on cost-effectiveness.

We will now argue for a new approach to the theory and practice of TCs, by focussing on recent research in the area of affect regulation, affiliative relating and the neurophysiology underpinning these. This area of rapid research development indicates new ways in which we can create opportunities for people to progress through relationships and potentially make radical changes in how our minds work (Davidson \& McEwen, 2012).

\section{EVOLUTIONARY APPROACHES TO 'THERAPEUTIC' COMMUNITIES}

Rather than approach therapy from a particular model, an alternative is to derive interventions from scientific studies of core psychological processes, and in particular from an understanding of the evolved systems of emotional processing and their regulation. Here, we describe a theoretical model for a third-generation TC that builds on the second generation. It is derived from research in child and social development and the neuroscience of affect regulation that views evolved, affiliative motivation as core to affect regulation. This facilitates the maturation of social cognitive abilities such as mentalizing and maintaining a competent sense of self, especially when occurring within a contextual behavioural approach. The reason for starting with the power of affiliative processes as core to therapeutic endeavours is that considerable research shows that loving and caring environments play major roles in the maturation of a whole range of emotional and cognitive abilities (Cozolino, 2007; Davidson \& McEwen, 2012) and even genetic expression (Belsky \& Pluess, 2009). The importance of the attachment relationship for subsequent developments of emotion regulation and social relationship was one of the crucial insights of John Bowlby $(1969,1973,1980)$.

As noted above, Bowlby argued that the early environment should offer two types of safeness: a safe haven that enables the child to return to a source of comfort and support in the face of distress and uncertainty and a safe base that provides the security and safeness to go and explore both inner and outer worlds, thus developing insight, understanding and the skills necessary for social living. Any therapy that is rooted in attachment theory must focus on these two provisions. Thus, to follow this approach, we need to understand the mechanisms by which threat and safeness operate. We contrast safeness and safety by suggesting that safety is threat focussed and involves stopping or getting away from threats-as in safety seeking. Even when 'out of harm', one's attention may still be vigilant to the possible return of threat. Safeness, however, depicts an open explorative attention and is focussed on slowing and also growing and developing. In safe environments (Bowlby's safe base), people take risks, can engage with potential frightening things and try new things; in threatening environments, people monitor both threat and their safety and are less creative and open (Gilbert, 1993).

\section{The Evolutionary Function Analysis of Emotion Systems}

Evolved social mentalities (e.g. attachment) are underpinned with evolved functional emotion systems. Recent research into the evolution of emotion suggests it is now possible to identify at least three evolved types of emotion regulation system, each with a different function and triggered in different contexts (Depue \& Morrone-Strupinsky, 2005). Stated briefly, these are the following:

1. Threat and self-protection-focussed system-this enables detection, attention processing and response to threats. There is a menu of threat-based emotions such as anger, anxiety and disgust and a menu of defensive behaviours such as fight, flight, submission and freeze.

2. Drive, seeking and acquisition-focussed system-this enables the individual to pay attention to advantageous resources. An experience of pleasure and achievement is associated with pursuing and securing them.

3. Contentment, soothing and affiliative-focussed systemthis is associated with a distinct positive affect of parasympathetic slowing. It is experienced as contentment, openness and peaceful well-being. It 
occurs when individuals are no longer threat focussed or seeking resources but are satisfied. These three systems are depicted in Figure 2.

A key to mental health is an ability to tolerate, blend balance and flexibly integrate the function of these systems. Mental health difficulties are often linked to people's direct effort to regulate threat by avoidance or engaging in safety-seeking behaviours (Gilbert, 1993). Many forms of anxiety disorder operate such that the short-term relief given by a safety-seeking behaviour (e.g. escape or compulsive washing) becomes reinforcing of the behaviour. Moreover, as noted above, one defensive-safety emotion (e.g. anger) or behaviour (e.g. rumination) can be a cover for avoiding another emotion (e.g. sadness). Sometimes, the drive system is used to regulate threat emotions, e.g. a need to do, have or achieve to feel secure from rejection, which can be unhelpful: excessive perfectionism, anorexia, gambling and workaholism are examples. The person's positive sense of self is contingent on frequent achievements.

However, all mammalian young are soothed by affiliation and comfort from (m)others. Operating though oxytocin and the parasympathetic autonomic nervous system and other systems, caring behaviour is well known to have soothing and threat-regulating effects on recipients (Depue \& Morrone-Strupinsky, 2005). Indeed, for most people, caring and affiliative relationships are the most important sources of threat regulation (Cacioppo \& Patrick, 2008). For the most part, threat and drive emotions stimulate the sympathetic nervous system, whereas soothing, calming and feeling safe are linked to the parasympathetic system, which in turn has a vast array of physiological and psychological effects (Porges, 2007). There is growing evidence that feeling socially safe, which is linked to parasympathetic activity, is a better predictor of vulnerability to psychopathology than 'excitement-based' positive emotion (Gilbert et al., 2008) and a better predictor than general positive or negative affect and social support (Kelly, Zuroff, Leybman, \& Gilbert, 2012).

So the evolution of 'caring' is one evolved root for compassion (Gilbert \& Choden, 2013). Compassion is commonly defined as 'a sensitivity to the suffering of self and others with a deep commitment to try to relieve and prevent it' (Gilbert \& Choden, 2013, p. 94). This kind of definition highlights two different but integrated mental sets or psychologies. The first is the ability to 'pay attention' to distress and difficulties, to notice them as they arise and to turn towards and be able to tolerate them, along with being able to empathize (mentalize) and make sense of them, in contrast to turning away, closing down, avoiding, blocking off, dissociating, denying and so forth. The second psychology is more action focussed and is concerned with acquiring and practising the skills necessary to address difficulties, which is partly linked to wisdom (Germer \& Siegel, 2012). So, for example, a client can become aware that part of their anger and mistrust is rooted in trauma memories. So using a compassionate approach, they first work on creating a sense of secure base and safe haven from which they can then begin to work with engaging with those memories (first psychology) and acquiring the skills and wisdom to be able to transform or re-script those memories (second psychology). Therapeutic benefits of training people in compassion have received increasing attention over the last decade. Indeed, there is now a range of compassion-focussed training approaches with increasing evidence of the value of compassion training (Hoffmann, Grossman, \& Hinton, 2011).
Driven, excited, vitality

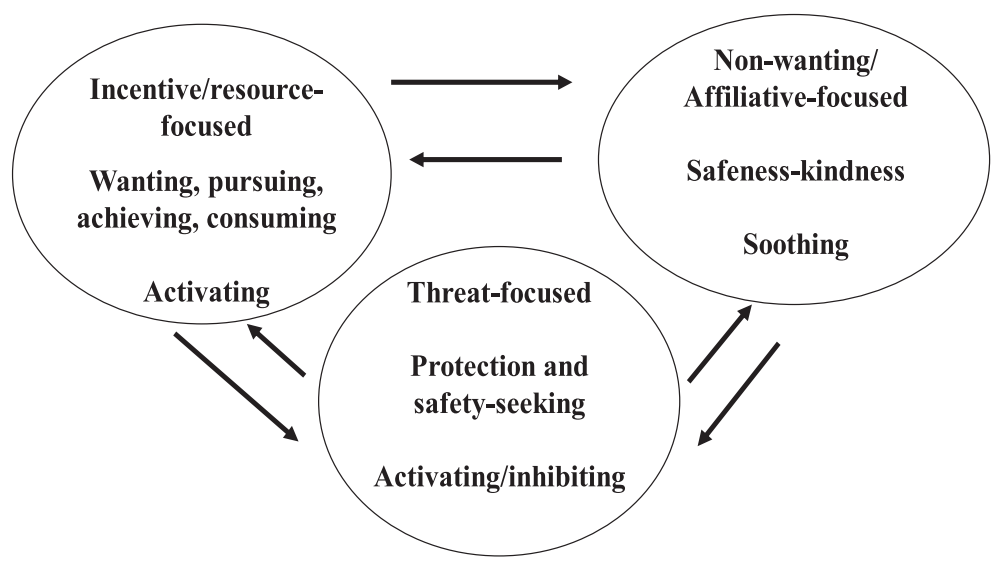

Anger, anxiety, disgust

Figure 2. Three types of affect regulation system 
Compassion can permeate the relationship to one's self as well as to others. Not only does affiliation and access to caring others facilitate soothing in the context of stress, but Bowlby also outlined how it enables courage to engage with acts that are frightening. Perhaps one of the clearest demonstrations of this is the visual cliff where infants are encouraged to cross the cliff even though they are clearly frightened: parental encouragement and a secure attachment enable them to cross. Hence, courage is influenced by access to affiliative others.

This perspective therefore suggests that TCs should first and foremost focus on creating safe and affiliative environments that facilitate courage and exploration (safe/secure base). Physiologically, the community would constantly try to shift from sympathetic dominant to parasympathetic engagement (Porges, 2007). We predict that stimulation of the parasympathetic autonomic nervous system within social contexts requires discouragement of high expressed emotion and shaming experiences. We do not mean this in a sense of avoidance because obviously learning to tolerate higher levels of emotional arousal is important for some individuals, but it should not be encouraged as something that is necessarily good. This is particularly important since individuals with mental health problems have highly sensitized threat systems and increased sympathetic tone with difficulties in calming down. They may find it difficult to avail themselves of affiliative relationships and utilize parasympathetic soothing systems. If a person is unable to use affiliative feelings, then they may be too reliant on trying to regulate the threat system with solutions from the threat or drive system. When individuals lack a secure base and safe haven and access to soothing others, regulation of threat-based emotion becomes very difficult. Such individuals can become preoccupied with their own internal world and intrapersonal process, cut off from the potential corrective input of empathic and compassionate others. Therapeutic interventions that focus only on teaching personal coping skills may inadvertently be reinforcing the idea that emotion regulation is something that goes on purely in one's own head-when in fact evolutionary mechanisms for affect regulation are very much interpersonal and social (Cacioppo \& Patrick, 2008).

\section{The Affiliative-Cooperative Environment}

Developing positive feelings is not just about being the recipient of care (which can be helpful) but also about feeling that the care we offer to others can be appreciated. To feel that we can be helpful and rewarding to others and make a contribution that is valuable and appreciated by others is core to developing a sense of self-value and well-being (Heard \& Lake, 1986) and a sense of belonging (Baumeister \& Leary, 1995). Gilbert (1984) suggested that part of the depressed person's experience of worthlessness is that they feel they do not matter to others, that they have little that others value or appreciate. Understanding others' goals and needs, followed by altruistic helping, has been detected in chimpanzees and young children and is rewarding in its own right (Warneken \& Tomasello, 2009). Developing an empathic concern for others and taking pleasure in their improvement are important aspects of social relating (Krill \& Platek, 2012). Indeed, as Yalom (1995) has highlighted many times, one of the therapeutic benefits of a group is that it provides opportunities to be validated and supported by others and also opportunities to be valuing and supporting of others and in that way develop a sense of one's value to others. This process of reciprocality can be central to a sense of belonging, affiliation and change. Bates (2005) wrote of the value of mutually shared compassion in group work with severe socially anxiety clients, noting how it contributed to a sense of being able to contribute and be helpful to others:

We have observed, both in our inpatient and outpatient groups that feedback, like mercy, is twice blessed. It is as much a gift for the giver as for the recipient. The experience of a group member having something to give another counters a pervasive sense of being no value to others. (Bates, 2005, p. 376)

Hence, creating opportunities for clients to help each other and form affiliative relationships on the basis of mutual helping is possibly one of the most important opportunities for learning new ways of relating and regulating emotions that a TC can provide. Indeed, whereas research into emotion regulation has typically looked at intrapersonal factors (personal cognitions, beliefs and ruminative processes), recent research is focussing on interpersonal process and style. For example, Niven, Macdonald, and Holman (2012) found that interpersonal styles linked to high anxious attachment, low empathic concern and low perspective taking were associated with poor social relationships and lower positive mood. Crocker and Canevello (2008) found that compassionate self-goals (such as wanting to be supportive of others) predict feelings of closeness and connectedness and increased social support and trust, whereas self-image goals (such as trying to convince others that you are right and avoiding shame) predict conflict, loneliness and feelings of fear and confusion. In the development of attachment theory approaches to psychotherapy, Fonagy and his colleagues have indicated the importance of mentalizing and the abilities of individuals to think about, be interested in and relate to the minds of others (Allen \& Fonagy, 2007). These key qualities are more likely to arise if someone feels safe, and the compassion system is stimulated. 
Many individuals who have complex psychological problems have come from backgrounds in which others were more likely to have been threats than sources of help (Liotti \& Gumley, 2008). Parents can send conflicting messages of safeness and threat, creating a complex confusion of approach-avoidance conflicts when it comes to giving help and being helped (Liotti \& Gilbert, 2011; Liotti \& Gumley, 2008). Under these conditions, the need to become self-sufficient and self-regulating becomes part of self-absorption and preoccupation.

The ability to contribute to others' improvement and their lives shifts attention out of self-focus. Sharing in common experiences of suffering and having a real desire to avoid shaming others can have important effects on oneself. Therefore, it is important to be open to the suffering of others, respond to them compassionately, offer help and develop empathic connectedness to other members of a community. Equally, it is important to develop and practise skills of self-compassion. We stress that affiliative relating is not a one-way street.

In summary, we have argued that in order for a TC to develop an environment of safeness and connectedness to others, we can turn to evolutionary, social and developmental psychology and neurophysiological systems for the underpinnings of safeness. Safeness can focus on the activation of compassionate motives and affiliative emotions, on helping participants think of their role in helping others and thus shift attention out of self-absorption and threat preoccupation, on providing opportunities for valuing and reinforcing interactions and on trying to give experiences of parasympathetic soothing (Gilbert \& Choden, 2013). There is increasing evidence that therapies that specifically focus on the development of compassion in participants produced important psychological change (Bates, 2005); several case series and single case studies have demonstrated the efficacy of developing compassion for oneself and others in a variety of clinical populations (Ashworth \& Clarke, 2012; Ashworth, Gracey, \& Gilbert, 2011; Mayhew \& Gilbert, 2008). A number of uncontrolled pilot trials of group compassion-focussed therapy have also been carried out (Gilbert \& Procter, 2006; Laithwaite et al., 2009). A recent controlled trial in recovery from psychosis showed that group-provided compassion-focussed therapy was significantly more helpful than treatment as usual on a range of measures (Braehler et al., 2012). Central to this was the observation that efforts to understand and help each other contributed to participant improvement. There is also increasing evidence that compassion-focussed and affiliative practices impact on a range of neurophysiological processes, including producing change in the frontal cortex and other affect regulation systems (e.g. Leiberg, Klimecki, \& Singer, 2011; Weng et al., 2013).

These motives for affiliative relating can be further advanced with clarification of the compassionate model.
This highlights the evolved difficulties we have in the regulation of emotion; the way the mind easily creates loops between thinking and feeling, which can be difficult to break out of; the fact that our social values are created in specific historical and social contexts; and the fact that we are all interdependent. This builds to an insight that much of what goes on in our minds, and certainly our mental health difficulties, is not our fault and that nonblaming insight provides a platform for the mutual taking of responsibility to help both self and others. Shaming and blaming however create defensive manoeuvres. However, developing compassionate approaches in a TC is not about anger or conflict avoidance. Indeed, compassion often builds strength for honest communication and engagement with painful issues.

There are two further theoretical models that we believe are of relevance in a new generation of TCs. The first of these is social learning theory and its application in FAP. The second is behavioural activation and the role of the drive system in a community.

\section{THE CONTRIBUTION OF SOCIAL LEARNING THEORY AND FUNCTIONAL ANALYTIC PSYCHOTHERAPY}

Social learning theory was developed by Bandura (1977). He suggested that human behaviour might be learned observationally through modelling, i.e. from observing others, which later serves as a guide for action. Thus, members who have been in the community longest may model behaviour to newer members. Bandura also described 'reciprocal determinism', i.e. behaviour influences environment and vice versa. For example, if members in a community avoid another member, it may confirm the beliefs of this member that others do not listen or care about them. It may persuade this member to spend more time alone and increase their expression of distress and challenging behaviour. This in turn confirms the group members' expectations that this member is not safe or that his or her behaviour is willful, and a vicious circle develops whereby the desire to avoid the member is further reinforced (Taylor \& Sambrook, 2012).

A related approach to social learning is FAP (Tsai et al., 2008). It is grounded in learning theory and is a functional analytic view of the therapeutic relationship and behaviour between a client and therapist. There is much evidence from learning theory for shaping behaviour in context and some evidence on how FAP can enhance outcomes when it is integrated in cognitive-behavioural therapy for depression or other disorders (Kohlenberg, Kanter, Bolling, \& Parker, 2002). The first principle of FAP is that a therapist has to be aware of interpersonal behaviours, assess their function and shape more effective interpersonal behaviours through reinforcement. Reinforcement is 'natural' rather than 
contrived: instead of using insincere praise or tokens, FAP asks therapists to share their emotional and cognitive responses to client behaviour, promoting closeness and engagement. In a TC, this translates into reinforcing acts of courage of one member by compassion from other members (Kanter J., personal communication). Members need to be adequately prepared for such an environment as they will be asked to speak the truth compassionately in the moment and tell others what they think, what they feel and what they need and to try to make a deep sense of connection with others even if it feels scary or risky. This can be emotionally quite intense. The rationale is that practising such behaviours in the moment enables a person to transfer these skills in the broader community and other people in their life. Unhelpful behaviours (e.g. self-harming, being withdrawn and seeking excessive reassurance) are if possible ignored, leading to extinction (unless the behaviour puts either himself or herself or other members at serious risk of harm when the aim must be to ensure psychological and physical safety). The behaviour may later be reflected on and understood in its evolutionary context with compassion. There is no punishment by shame even for unacceptable behaviours such as bullying. Behaviours that involve courage and promote affiliation are naturally reinforced by compassion. Members would try to avoid positive reinforcement of behaviours that are unhelpful (e.g. 'accommodating' behaviour, such as helping someone to avoid a fear by doing a task for them or being overprotective). The principles of awareness, courage and compassion complement and enhance compassion-focussed therapy as a core component of an effective TC. Moreover, it creates the environmental contexts that enable people to feel safe, understood and orientated to developing compassionate interactions and compassionate ways of dealing with their own and others' suffering.

An important component of reinforcement is that the closer in time and place the behaviour is to its consequences, the greater the effect of those consequences. Thus, it is important to be aware and attentive and to respond at the time that a movement towards an act of courage occurs. Because members of a TC are living together, the principles of FAP can be extended to the relationships between members and staff. It means that reinforcement does not have to happen only in individual or group therapy time, but more importantly also in the times between therapy sessions and in everyday interactions between clients, between clients and staff and between staff. Thus, it is important for members to be aware of what would be each other's acts of courage and have the skills to understand the motivation of unhelpful behaviours. They may then respond compassionately and look out for any effort towards change in the moment. Although skilled therapists may be trained to deliver FAP, what is not known is the effectiveness of training members of TC in an adapted or 'light' version of FAP. This needs to be tested empirically. The theory is that a TC requires a culture of positive reinforcement for acts of courage, especially in helping others to engage with things they are fearful of and to face their own feared or avoided areas. These arise and are delivered with an understanding of the components of compassion such as distress tolerance and empathy. There may be a formal FAP group or an emphasis on awareness to look out for in other members. Again, it is crucial for members to have in mind each client's formulation specifying unhelpful behaviours that may show up in the community and acts of courage that need to be positively reinforced to assist change. In compassion-focussed approaches, acts of courage are learning the affiliative support of others, being open with oneself and learning to treat oneself more kindly and dropping shaming and blaming of self or others while taking responsibility for change. Any movement towards taking responsibility for trying to help oneself for others should therefore be positively reinforced in the moment with compassion and appropriate affiliative (especially non-verbal) behaviour and engagement.

An important feature of a social learning environment is a focus on goals as a means towards acting on one's values. The goals may relate to the presenting problems or to interpersonal behaviour and relating to others. Progress towards goals needs to be monitored regularly on appropriate rating scales with feedback provided. Current TCs believe that it is important for members to be involved in a structured activity of social behaviours, which is in keeping with the principle of enablement and rehabilitation to society. A good therapeutic environment will have a range of opportunities in the form of occupational therapy, social events and everyday activities such as cooking, cleaning, gardening and volunteering in the wider community. It could also include aspects of a retreat with mindfulness meditation and compassionate imagery exercises. Activity can also assist in building capacity for mentalizing-the capacity to empathize with and understand the perspectives of other members by the process of socializing and working with others (another component of compassion-focussed approaches). However, some members may benefit from a more systematic assessment and skills-based teaching of behavioural activation (Dimidjian et al., 2006; Martell, Dimidjian, \& Herman-Dunn, 2010). For example, a functional assessment of activity will allow a member to have a good understanding of the contingencies (antecedents and consequences) of the behaviour that is maintaining their mood and to then plan to act against the way they feel in keeping with their values. However, a successful environment will provide a diverse and stable range of reinforcers of members who respond 'in the moment' when an individual moves towards their goal. 


\section{A THIRD-GENERATION THERAPEUTIC COMMUNITY IN PRACTICE}

We started with the premise that living in a community may confer therapeutic advantages over individual outpatient therapy and should be investigated further. Currently, however, we suggest that some of the processes in psychodynamically informed communities lack an evidence base. In so far as some communities encourage the expression of high emotion, they may be counterproductive. In contrast, recent research has shown that the development of affiliative relations are central to the ability to engage with feared and avoided emotions and difficulties. These therefore should be the priority, focussing on the processes that will mediate these factors. In practice, this will include the following:

1. Using an evolutionary model to inform members of how tricky the human brain is, which provides a basis for de-shaming and blaming and a sense of common humanity in that we are all on the same life's journey

2. To provide insight into the nature of human emotion regulation and in particular the importance of affiliative emotion systems as threat regulators, the sources of positive emotion well-being and meaning and therefore the value of gaining access and stimulating that system

3. Clarifying the nature of compassion and dispelling myths about compassion such as its being related to weakness or something one does not deserve

4. Provide the interpersonal basis for a secure base and safe haven, which facilitates the ability to tolerate, empathize and understand distress.

5. From this affiliative context, to stimulate the motivation to engage with painful and difficult things and promote the courage to act in ways that are consistent with the member's therapeutic goals and personal values

6. Opening up to being able to be sensitive and attentive to the distress and therapeutic goals of others with an interest in helping people to achieve their goals-hence enabling the community to operate by supporting each other's therapeutic journey and building a sense of community from that process

7. Respond to acts of courage in others and provide compassionate ways of understanding the source, nature and ways of resolving and being honest about conflicts

Our hypothesis is that when compassionate values and goals are carefully explained and placed at the heart of a community and how and why they are rooted in the evolutionary understanding that the human brain is very tricky and easily thrown into threat processing, this provides a context of change that is different from the current TC approaches.
We now summarize the principles of a third-generation $\mathrm{TC}$ in action. The emphasis is on interpersonal behaviour and mediating processes rather than particular structures or groups. The principles build on second-generation TCs and can also be applied to psychologically informed environments and inpatient settings (especially in longterm rehabilitation units).

1. Members of a community would have a good psychological understanding (with a compassionfocussed and contextual behavioural formulation) of their own difficulties. The emphasis would be on the context of their interpersonal behaviour and relating to others and the development of their problems. Members would share this formulation so that other members would be aware of how their problem will 'show up' in the community and what behaviours they need to look out for and how these relate to their goals.

2. Members would learn and practise compassionfocussed approaches to their self and others so that the culture supports being motivated and attentive to each other's needs, empathic, respectful, sympathetic, kind, accepting, non-judgemental and tolerant of each other's distress. Part of this will involve the use of mindfulness and compassionate imagery practices. This is an environment of relatively low expressed emotion and trying to prevent unnecessary activation of the threat system. This involves increasing awareness of the impact of one's behaviour on others in a non-shaming way (e.g. "Today I'd like to take more of an interest in "Sally" and spend a little time really finding out a bit more about her and how I can be helpful to her while she would like me to be helpful to her').

3. Members would be taught to increase awareness by focussing their attention not only on their own thoughts, feelings and memories, and values and bodily sensations but also on how other contingencies affect them, how their own behaviour affects others and understanding the context with the goals of other members. Awareness is therefore paying attention in the moment without judgement and being aware of one's goals and values and acts of courage in others. Such exercises include eyes-closed meditation, eyes-on and interpersonal meditations and listening and walking meditations.

4. Members would positively reinforce acts of courage in one another. Such acts include being honest, doing vulnerable disclosures, being authentic, discussing losses, discussing values and what the person stands for, taking risks, confronting difficult situations or doing exposure and behavioural experiments to test out one's fears and expectations depending on the presenting problem. When 
members are aware of such acts, they can try to respond 'naturally' and compassionately in the moment when another member makes an effort to change. Opening up to others also means taking joy in their successes. Helping people pay attention to these issues will over time help them begin to feel pleasure in the successes of others rather than being envious or totally closed in and only interested in themselves. We acknowledge that efforts to change in others can be difficult to be aware of in a community, and remembering different presentations in other members can also be difficult. This is why it is so important to increase such awareness by telling others what to look out for. Awareness of planned acts of courage for the day can be put up as reminders for others on a physical or digital notice board and discussed at a daily planning meeting. ('Today I will test out my fears of being criticized by generating a kind of voice in my mind when I start to get the anxious about speaking up in a community meeting. I'm going to try to focus my attention externally and notice and look for facial expressions of support and others'.) Acts of courage would also be reflected on in community meetings and reinforced by others, and a log of the courageous acts that have been taken should be kept.

5. Members would use the principles of 'extinction' by generally ignoring or walking away from the unhelpful behaviours of others unless it puts a member at physical risk. They would refrain from using 'punishment' by shaming or by accommodating one another's unhelpful behaviours. Unhelpful behaviours would be reflected on and understood in a compassion-focussed model. Unhelpful behaviours may be shared in a small group or as a written formulation between all members, so they are aware of how they show up in the community.

6. Staff need to feel supported and safe with themselves and preferably are affiliative with each other. Staff who have not resolved conflicts among themselves are not in a position to provide safe and affiliative environments. Furthermore, staff need to model being authentic, fully present, compassionate and caring with colleagues and members. Staff will need an external supervisor who can use compassionfocussed and functional analytical models. One of the most important processes for staff is to understand the model themselves and not engage in splitting, i.e. when members act to try to keep themselves safe by focussing on one member to suggest that 'they shouldn't be here'.

7. Members could support one another to reflect, to devise behavioural experiments to test out their beliefs, to consider an alternative understanding of their problem or to develop a more functional way of responding. Members are expected to care for and emotionally support one another and to be 'held' in mind by other members as part of a compassionate approach. Members can be encouraged to enquire about and support one another's acts of courage and kindness to others. A culture of connectedness with others will therefore evolve naturally and can be facilitated by formal structures for joining and leaving and regular meetings. There would be an option for calling 'crisis meetings' when a member can obtain support from others. Members of nonresidential TCs can phone or visit another member of the community so long as this can be carried out safely and with mutual benefit and awareness by the staff.

8. Members would be learning to communicate openly, honestly and naturally with one another and to validate each other when they are distressed. For some, this requires a skills-based approach that helps to recognize different emotions and to respond in less harmful ways.

9. Members would have a programme of structured activity and a timetable for achieving their goals that is facilitated by a diverse and stable range of positive reinforcers in the environment. They would have the option of skills-based learning for rehabilitation so that the community would facilitate individuals to reach their potential and return to employment.

10. There would be a culture of clearly defined goals in terms of acts of courage for individual members and the community, relating to interpersonal behaviour as well as presenting problems. The goals should be regularly monitored and feedback on progress provided to the member.

11. There could be a culture of as much democracy and informality as possible within the community depending on the context. However, democracy and influence should support one or more of the factors above and be incorporated as a strategy in individual formulations.

12. Boundaries or rules are developed by members and motivated by the need to feel safe and connected with one another. The function behind boundary setting needs to be part of the process of the community. However, the setting of boundaries and rules is limited by their context (e.g. in forensic or hospital settings where institutions and the staff have certain boundaries in place).

13. There would a culture of empiricism so that members are encouraged to participate in process and outcome research to determine which factors in the environment promote and mediate therapeutic change. 


\section{Future Research}

The research question is whether our proposed therapeutic environment can either enhance the delivery of evidence-based therapies delivered in these contexts or be powerful enough without any additional therapies. First, the research will need to be on the feasibility, acceptability and process of change. If this is positive, then for costeffective reasons, entry to a TC will probably be in the context of stepped care and designed for those with the highest need or severity or whose treatment at an earlier step has failed. We suggest that what is needed is an RCT of clinical and cost-effectiveness that compares our thirdgeneration TC against the following: (i) one based on group psychodynamic therapy and (ii) treatment as usual, which be an evidence-based therapy delivered on a continuing outpatient basis, e.g. in persons with borderline personality disorder who have failed dialectical behaviour therapy (Linehan et al., 2006) or mentalization therapy (Bateman \& Fonagy, 2009) or a schema-focussed therapy (Giesen-Bloo et al., 2006). Lastly, there is need for more process research to influence the delivery of a TC. We need to understand more about the mediating factors (e.g. how best to enhance feeling safe or connected to others) that might facilitate change in a community. This would then be translated into monitoring the processes within a community to determine if the community is adhering to its model and the mediating factors in change.

\section{CONCLUSIONS}

Humans evolved in close-knit communities, and therefore, our brains are highly focused on and influenced by the quality of our personal relationships. This makes them ideal as therapeutic levers. To date, however, the use of the community as a therapeutic agent is primarily orientated by psychodynamic theory of uncertain evidence. In this paper, we have suggested to start again by looking at the evidence of how relationships work and in particular how they operate in various emotional and neurophysiological systems. There is increasing evidence that both compassion-focused and social learning approaches hold significant promise as therapeutic agents (Hoffmann et al., 2011). To date, however, this has not been extended into TC, and to us, this opens up an important avenue for research. An environment is built on affiliative relationships and compassion in response to acts of courage rather than 'reality confrontation' or catharsis. This may more readily foster change, because members are more open to feedback from others, feel safe to try out new behaviours, are mutually encouraging to do so and come to recognize they can play an active part in somebody's recovery journey, all of which increases a sense of belonging. The model places at its core the positive reinforcement of acts of courage, the experience of being valued and supported and also the experience of joy from recognizing oneself as helpful and supportive of others. Although it is true that we need to be able to tolerate and work appropriately with our threat-based emotions of anger and anxiety, what actually creates meaning and value in life is a sense of being valued and valuing, i.e. a sense of connectedness with and to others.

\section{ACKNOWLEDGEMENTS}

We would like to acknowledge the helpful discussions with Jonathan Kanter and Glenn Callaghan. D. V. receives salary support from the National Institute for Health Research (NIHR) Biomedical Research Centre for Mental Health at South London and Maudsley NHS Foundation Trust and the Institute of Psychiatry, King's College London. The views expressed are those of the authors and not necessarily those of the NHS, the NIHR or the Department of Health.

\section{REFERENCES}

Allen, J. G., \& Fonagy, P. (Eds.). (2007). Handbook of mentalization-based treatment. Chichester: Wiley.

Ashworth, F., \& Clarke, A. (2012). 'Learning to be compassionate to my tricky brain': A case illustration of the compassionate mind approach after stroke. Brain Injury, 26, 718-719. DOI: 10.3109/02699052.2012.660091

Ashworth, F., Gracey, F., \& Gilbert, P. (2011). Compassion focused therapy after traumatic brain injury: Theoretical foundations and a case illustration. Brain Impairment, 12, 128-139. DOI: 10.1375/brim.12.2.128

Ballatt, J., \& Campling, P. (2011). Intelligent kindness: Reforming the culture of health care. London: Royal College of Psychiatry Publications.

Bandura, A. (1977). Social learning theory. New York: General Learning Press.

Bateman, A., \& Fonagy, P. (2009). Randomized controlled trial of outpatient mentalization-based treatment versus structured clinical management for borderline personality disorder. American Journal of Psychiatry, 166, 1355-1364. DOI: 10.1176/ appi.ajp.2009.09040539

Bates, T. (2005). The expression of compassion in group cognitive therapy. In P. Gilbert (Ed.), Compassion: Conceptualisations, research and use in psychotherapy (pp. 369-386). London: Routledge.

Baumeister, R. F., \& Leary, M. R. (1995). The need to belong-Desire for interpersonal attachments as a fundamental humanmotivation. Psychological Bulletin, 117, 497-529. DOI: 10.1037/ 0033-2909.117.3.497

Belsky, J., \& Pluess, M. (2009). Beyond diathesis stress: Differential susceptibility to environmental influences. Psychological Bulletin, 135, 885-908. DOI: 10.1037/a0017376

Borthwick, A., Holman, C., Kennard, D., Mcfetridge, M., Messruther, K., \& Wilkes, J. (2001). The relevance of moral treatment to contemporary mental health care. Journal of Mental Health, 10, 427-439. DOI: 10.1080/09638230124277 
Bowlby, J. (1969). Attachment: Attachment and loss (Vol. 1). London: Hogarth Press.

Bowlby, J. (1973). Separation, anxiety and anger. Attachment and loss (Vol. 2). London: Hogarth Press.

Bowlby, J. (1980). Loss: Sadness and depression. Attachment and loss (Vol. 3). London: Hogarth Press.

Braehler, C., Gumley, A., Harper, J., Wallace, S., Norrie, J., \& Gilbert, P. (2012). Exploring change processes in compassion focused therapy in psychosis: Results of a feasibility randomized controlled trial. British Journal of Clinical Psychology, 52, 199-214. DOI: $10.1111 /$ bjc.12009

Cacioppo, J. T., \& Patrick, B. (2008). Loneliness: Human nature and the need for social connection. New York: Norton.

Campling, P., \& Haigh, R. (Eds.). (1999). Therapeutic communities: Past, present and future. London: Jessica Kingsley.

Cozolino, L. (2007). The neuroscience of human relationships: Attachment and the developing brain. New York: Norton.

Craft, M., Stephenson, G., \& Granger, C. (1964). A controlled trial of authoritarian and self-governing regimes with adolescent psychopaths. American Journal of Orthopsychiatry, 34, 543-554. DOI: $10.1111 /$ j.1939-0025.1964.tb02224.x

Crocker, J., \& Canevello, A. (2008). Creating and undermining social support in communal relationships: The role of compassionate and self-image goals. Journal of Personality and Social Psychology, 95, 555-575.

Danquah, A. N., \& Berry, K. (2013). Attachment theory in adult mental health: A guide to clinical practice. London: Routledge.

Davidson, R. J., \& McEwen, B. S. (2012). Social influences on neuroplasticity: Stress and interventions to promote wellbeing. Nature Neuroscience, 15, 689-695. DOI: 10.1038/nn.3093

De Leon, D. (2000). The therapeutic community: Theory, model and method. New York: Springer.

Dearing, R. L., \& Tangney, J. P. (Eds.). (2011). Shame in the therapy hour. Washington: American Psychological Society.

Depue, R. A., \& Morrone-Strupinsky, J. V. (2005). A neurobehavioral model of affiliative bonding: Implications for conceptualizing a human trait of affiliation. Behavioral and Brain Sciences, 28, 313-321. DOI: 10.1017/S0140525X05000063

Dimidjian, S., Hollon, S. D., Dobson, K. S., Schmaling, K. B., Kohlenberg, R. J., Addis, M. E., ... Jacobson, N. S. (2006). Randomized trial of behavioral activation, cognitive therapy, and antidepressant medication in the acute treatment of adults with major depression. Journal of Consulting and Clinical Psychology, 74, 658-670. DOI: 10.1037/0022-006x.74.4.658

Dunbar, R. I. M. (2007). Mind the bonding gap: Or why humans aren't just great apes. Proceedings of the British Academy, 154, 403-433.

Dunbar, R. I. M. (2010). The social role of touch in humans and primates: Behavioural function and neurobiological mechanisms. Neuroscience and Biobehavioral Reviews, 34, 260-268. DOI: 10.1016/j.neubiorev.2008.07.001

Dunbar, R. I. M., \& Barrett, L. (2007). The Oxford handbook of evolutionary psychology. Oxford: Oxford University Press.

Fenney, B. C., \& Thrush, R. L. (2010). Relationship influences on exploration in adulthood: The characteristics and function of a secure base. Journal of Personality and Social Psychology, 98, 57-76. DOI: $10.1037 / \mathrm{a} 0016961$

Fiestas, F., \& Ponce, J. (2012). Efficacy of the therapeutic community model in the treatment of drug use-related problems: A systematic review. Revista Peruana de Medicina Experimental y Salud Pública, 29, 12-20. DOI: 10.1590/S1726-46342012000100003
Germer, C. K., \& Siegel, R. D. (2012). Wisdom and compassion in psychotherapy. New York: Guilford.

Giesen-Bloo, J., van Dyck, R., Spinhoven, P., van Tilburg, W., Dirksen, C., van Asselt, T., ... Arntz, A. (2006). Outpatient psychotherapy for borderline personality disorder-Randomized trial of schema-focused therapy vs transference-focused psychotherapy. Archives of General Psychiatry, 63, 649-658. DOI: 10.1001/archpsyc.63.6.649

Gilbert, P. (1984). Depression: From psychology to brain state. London: Lawrence Erlbaum Associates.

Gilbert, P. (1993). Defence and safety: Their function in social behaviour and psychopathology. British Journal of Clinical Psychology, 32, 131-153. DOI: 10.1111/j.2044-8260.1993. tb01039.x

Gilbert, P. (2005). Compassion and cruelty: A biopsychosocial approach. In P. Gilbert (Ed.), Compassion: Conceptualisations, research and use in psychotherapy (pp. 3-74). London: Routledge.

Gilbert, P. (2007). The evolution of shame as a marker for relationship security. In J. L. Tracy, R. W. Robins, \& J. P. Tangney (Eds.), The self-conscious emotions: Theory and research (pp. 283-309). New York: Guilford.

Gilbert, P. (2009). The compassionate mind. London: ConstableRobinson.

Gilbert, P. (2010). An introduction to compassion focused therapy in cognitive behavior therapy. International Journal of Cognitive Therapy, 3, 97-112. DOI: 10.1521/ijct.2010.3.2.97

Gilbert, P. (2011). Shame in psychotherapy and the role of compassion focused therapy. In R. L. Dearing, \& J. P. Tangney (Eds.), Shame in the therapy hour (pp. 325-354). Washington: American Psychological Society.

Gilbert, P., \& Choden, K. (2013). Mindful compassion. London: Robinson.

Gilbert, P., \& Procter, S. (2006). Compassionate mind training for people with high shame and self-criticism: Overview and pilot study of a group therapy approach. Clinical Psychology $\mathcal{E}$ Psychotherapy, 13, 353-379. DOI: 10.1002/cpp.507

Gilbert, P., McEwan, K., Mitra, R., Franks, L., Richter, A., \& Rockliff, H. (2008). Feeling safe and content: A specific affect regulation system? Relationship to depression, anxiety, stress and self-criticism. Journal of Positive Psychology, 3, 182-191. DOI: $10.1080 / 17439760801999461$

Gilmore, D. D. (1990). Manhood in the making: Cultural concepts of masculinity. Connecticut: Yale University Press.

Haigh, R. (1999). The quintessence of a therapeutic community. In P. Campling, \& R. Haigh (Eds.), Therapeutic communities: Past, present and future (pp. 246-257). London: Jessica Kingsley.

Hansen, J. T., \& Slevin, C. (1996). The implementation of therapeutic community principles in acute care psychiatric hospital settings: An empirical analysis and recommendations to clinicians. Journal of Clinical Psychology, 52, 673-678. DOI: 10.1002/(sici)1097-4679(199611)52:6<673::aid-jclp9>3.0.co;2-1

Heard, D. H., \& Lake, B. (1986). The attachment dynamic in adult life. British Journal of Psychiatry, 149, 430-438. DOI: 10.1192/ bjp.149.4.430

Hoffmann, S. G., Grossman, P., \& Hinton D. E. (2011). Lovingkindness and compassion meditation: Potential for psychological intervention. Clinical Psychology Review, 13, 1126-1132. DOI: 10.1016/j.cpr.2011.07.003

Holt-Lunstad, J., Smith, T. B., \& Layton, J. B. (2010). Social relationships and mortality risk: A meta-analytic review. PLOS Medicine, 7. DOI: 10.1371/journal.pmed.1000316 
Jones, M. (1956). The concept of a therapeutic-community. American Journal of Psychiatry, 112(8), 647-650.

Kelly, A. C., Zuroff, D. C., Leybman, M. J., \& Gilbert, P. (2012). Social safeness, received social support, and maladjustment: Testing a tripartite model of affect regulation. Cognitive Therapy and Research, 36, 815-826. DOI: 10.1007/ s10608-011-9432-5

Kelman, H. C., \& Hamilton, V. L. (1989). Crimes of obedience: Toward a social psychology of authority and responsibility. New Haven, CT: Yale University Press.

Kennard, D. (1998). An introduction to therapeutic communities. London: Jessica Kingsley.

Kohlenberg, R. J., Kanter, J. W., Bolling, M. Y., \& Parker, C. R. (2002). Enhancing cognitive therapy for depression with functional analytic psychotherapy: Treatment guidelines and empirical findings. Cognitive and Behavioral Practice, 9, 213-229. DOI: 10.1016/s1077-7229(02)80051-7

Krill, A. L., \& Platek, S. M. (2012). Working together may be better: Activation of reward centers during a cooperative maze task. PLoS One, 7, e30613-e30613. DOI: 10.1371/journal. pone. 0030613

Laithwaite, H., O'Hanlon, M., Collins, P., Doyle, P., Abraham, L., Porter, S., \& Gumley, A. (2009). Recovery after psychosis (RAP): A compassion focused programme for individuals residing in high security settings. Behavioural and Cognitive Psychotherapy, 37, 511-526. DOI: 10.1017/ s1352465809990233

Lees, J., Manning, N., \& Rawlings, B. (2004). A culture of enquiry: Research evidence and the therapeutic community. Psychiatric Quarterly, 75(3), 279-294. DOI: 10.1023/B:PSAQ.000003 1797.74295.f8

Lehman, A., \& Ritzler, B. (1976). Therapeutic-community inpatient ward-Does it really work. Comprehensive Psychiatry, 17, 755-761. DOI: 10.1016/0010-440x(76)90023-7

Leiberg, S., Klimecki, O., \& Singer, T. (2011). Short-term compassion training increases prosocial behavior in a newly developed prosocial game. PLoS One, 6(3), e17798. DOI: 10.1371/journal.pone.0017798

Li, S. C. (2003). Biocultural orchestration of developmental plasticity across levels: The interplay of biology and culture in shaping the mind and behavior across the life span. Psychological Bulletin, 129, 171-194. DOI: 10.1037/00332909.129.2.171

Linehan, M. M., Comtois, K. A., Murray, A. M., Brown, M. Z., Gallop, R. J., Heard, H. L., ... Lindenboim, N. (2006). Two-year randomized controlled trial and follow-up of dialectical behavior therapy vs therapy by experts for suicidal behaviors and borderline personality disorder. Archives of General Psychiatry, 63, 757-766. DOI: 10.1001/archpsyc.63.7.757

Liotti, G., \& Gilbert, P. (2011). Mentalizing, motivations and social mentalities: Theoretical considerations and implications for psychotherapy. Psychology and Psychotherapy, 84, 9-25. DOI: $10.1348 / 147608310 \times 520094$

Liotti, G., \& Gumley, A. (2008). An attachment perspective on schizophrenia: The role of disorganized attachment, dissociation and mentalization. In A. Moskowitz, I. Schafe, \& M. J. Dorahy (Eds.), Psychosis, trauma and dissociation (pp. 117-133). Chichester: Wiley.

Main, T. (1946). The hospital as a therapeutic institution. Bulletin of the Menninger Clinic, 10, 66-70.

Martell, C. R., Dimidjian, S., \& Herman-Dunn, R. (2010). Behavioral activation for depression: A clinician's guide. New York: Guilford Press.
Mayhew, S. L., \& Gilbert, P. (2008). Compassionate mind training with people who hear malevolent voices: A case series report. Clinical Psychology \& Psychotherapy, 15, 113-138. DOI: 10.1002/cpp.566

Mikulincer, M., \& Shaver, P. R. (2007). Attachment in adulthood: Structure, dynamics, and change. New York: Guilford.

National Institute for Health \& Clinical Excellence. (2009). Borderline personality disorder: The NICE guidelines on treatment and management [CG78]. London: National Institute for Health and Clinical Excellence.

Niven, K., Macdonald, I., \& Holman, D. (2012). You spin me right round: Cross-relationship variability in interpersonal emotion regulation. Frontiers in Psychology, 3, 394-405. DOI: 10.3389/ fpsyg.2012.00394

Pearce, S., \& Pickard, H. (2013). How therapeutic communities work: Specific factors related to positive outcome. International Journal of Social Psychiatry, 59, 636-645. DOI: 10.1177/ 0020764012450992

Perry, A. E., Darwin, Z., Godfrey, C., McDougall, C., Lunn, J., Glanville, J., \& Coulton, S. (2009). The effectiveness of interventions for drug-using offenders in the courts, secure establishments and the community: A systematic review. Substance Use and Misuse, 44, 374-400. DOI: 10.1080/10826080802347560

Porges, S. W. (2007). The polyvagal perspective. Biological Psychology, 74, 116-143. DOI: 10.1016/j.biopsycho.2006.06.009

Porter, R. (2002). Madness: A brief history. Oxford: Oxford University Press.

Rapoport, R. N. (1960) Community as doctor. London: Tavistock.

Roberts, J. (1997). How to recognise a therapeutic community. Prison Service Journal, 111, 4-7.

Royal College of Psychiatrists. (2010). The community of communities. Retrieved May 2012, from http:/ / www.rcpsych.ac.uk/ quality/qualityandaccreditation/therapeuticcommunities / communityofcommunities1.aspx

Sacks, J. Y., McKendrick, K., \& Hamilton, Z. (2012). A randomized clinical trial of a therapeutic community treatment for female inmates: Outcomes at 6 and 12 months after prison release. Journal of Addictive Diseases, 31, 258-269. DOI: 10.1080/10550887.2012.694601

Smith, L. A., Gates, S., \& Foxcroft, D. (2006). Therapeutic communities for substance related disorder. Cochrane Database of Systematic Reviews. DOI: 10.1002/14651858.CD005338.pub2

Tangney, J. P., \& Dearing, R. L. (2002). Shame and guilt. New York: Guilford Press.

Tangney, J. P., Wagner, P. E., Hill-Barlow, D., Marschall, D. E., \& Gramzow, R. (1996). Relation of shame and guilt to constructive versus destructive responses to anger across the lifespan. Journal of Personality and Social Psychology, 70, 797-809. DOI: 10.1037/0022-3514.70.4.797

Taylor, S. E. (2006). Tend and befriend biobehavioral bases of affiliation under stress. Current Directions in Psychological Science, 15, 273-277. DOI: 10.1111/j.1467-8721.2006.00451.x

Taylor, K. N., \& Sambrook, S. (2012). CBT for culture change: Formulating teams to improve patient care. Behavioural and Cognitive Psychotherapy, 40, 496-503. DOI: 10.1017/ s1352465812000069.

Tsai, M., Kohlenberg, R. J., Kanter, J. W., Kohlenberg, B., Follette, W. C., \& Callaghan, G. M. (2008). A guide to functional analytic psychotherapy: Using awareness, courage, love and behaviorism. New York: Springer.

Tucker, S. (1999). Community care: The therapeutic approach and learning to care. In P. Campling, \& R. Haigh (Eds.), Therapeutic communities: Past, present and future (pp. 151-161). London: Jessica Kingsley. 
Tung, J., Barreiro, L. B., Johnson, Z. P., Hansen, K. D., Michopoulos, V., Toufexis, D., ... Gilad, Y. (2012). Social environment is associated with gene regulatory variation in the rhesus macaque immune system. Proceedings of the National Academy of Sciences of the United States of America, 109, 6490-6495. DOI: 10.1073/pnas.1202734109

Van der Hart, O., Steele, K., \& Nijenhuis, E. (2006). The haunted self: Structural dissociation and treatment of chronic traumatization. New York: Norton.

Wallin, D. J. (2007). Attachment in psychotherapy. New York: Guilford.

Warneken, F., \& Tomasello, M. (2009). The roots of human altruism. British Journal of Psychology, 100, 455-471. DOI: 10.1348/000712608x379061
Weng, H. Y., Fox A. S., Shackman, A. J., Stodola, D. E., Caldwell, J. Z. K., Olson, M. C., ... Davidson, R. J. (2013). Compassion training alters altruism and neural responses to suffering. Psychological Science, 24, 1171-1180. DOI: 10.1177/ 0956797612469537

Whiteley, S. (2004). The evolution of the therapeutic community. Psychiatric Quarterly, 75, 233-248. DOI: 10.1023/B: PSAQ.0000031794.82674.e8

Yalom, I. D. (1995). The theory and practice of group psychotherapy. New York: Basic Books.

Zehr, H. (2002). Restorative justice. PA: Good Books.

Zimbardo, P. (2008). The Lucifer effect: How good people turn evil. London: Rider. 